\title{
Interferon- $\gamma$ enhances phorbol myristate acetate-induced cell attachment and tumor necrosis factor production via the NF-кB pathway in THP-1 human monocytic cells
}

\author{
YUICHI KURIHARA and MASUTAKA FURUE
}

\author{
Department of Dermatology, Graduate School of Medical Sciences, Kyushu University, Higashi-ku, Fukuoka 812-8582, Japan
}

Received January 5, 2013; Accepted April 2, 2013

DOI: $10.3892 / \mathrm{mmr} .2013 .1419$

\begin{abstract}
During inflammation, activated macrophages express adhesion molecules and produce cytokines that interact with other hematopoietic and stromal cells. THP-1 non-adherent human monocytic cells differentiate into plasticadherent macrophages via $\alpha_{\mathrm{V}} \beta_{3}$ integrin, by ERK activation in the presence of phorbol myristate acetate (PMA). This has proven to be a valuable model for investigating functional monocyte/macrophage diversity. Interferon- $\gamma($ IFN- $\gamma$ ) is a Th1-cytokine that is crucial in macrophage activation. In this study, we investigated the effects of IFN- $\gamma$ on adhesion and the secretion of tumor necrosis factor (TNF) by PMA-stimulated THP-1 cells. IFN- $\gamma$ is incapable of inducing cell attachment and TNF production; however, it cumulatively upregulated PMA-induced basal adhesion and TNF production. IFN- $\gamma$ increased $\alpha_{\mathrm{V}}$ integrin, ICAM-1 and VCAM-1 expression and among these PMA-induced cell surface adhesion molecules, the blocking antibody for $\alpha_{\mathrm{V}}$ integrin suppressed adhesion and TNF production. Furthermore, IFN- $\gamma$ enhanced PMA-induced NF- $\mathrm{B}$ phosphorylation and not ERK phosphorylation. Accordingly, the NF- $\kappa \mathrm{B}$ pathway inhibitor (BAY 11-7082) inhibited the enhancing effect of IFN- $\gamma$ on adhesion and TNF production. By contrast, the MEK inhibitor (U0126) almost completely eliminated PMA-induced basal adhesion and TNF production. In conclusion, IFN- $\gamma$ regulates macrophage activation by mediating the $\mathrm{NF}-\kappa \mathrm{B}$ signaling pathway.
\end{abstract}

\section{Introduction}

Inflammation is a fundamental process involved in protection against infection or injury (1). Macrophages are involved in

Correspondence to: Dr Yuichi Kurihara, Department of Dermatology, Graduate School of Medical Sciences, Kyushu University, 311 Maidashi, Higashi-ku, Fukuoka 812-8582, Japan E-mail: y-kuri@dermatol.med.kyushu-u.ac.jp

Key words: macrophage, adhesion, tumor necrosis factor, interferon- $\gamma, \mathrm{NF}-\kappa \mathrm{B}$ pathway acute and chronic inflammation $(2,3)$ and play multidirectional roles in the integrity of the immune system (4).

Interferon- $\gamma($ IFN- $\gamma)$ is important for macrophage activation during the innate and acquired immune response (5). Activated macrophages obtain increased cytokine production and enhanced tumoricidal and microbicidal function $(6,7)$. In various inflammatory and granulomatous conditions, IFN- $\gamma$ is critical in enhancing and sustaining macrophage activation $(8,9)$. However, its precise mechanism remains largely unknown and elucidating its role is essential in order to gain further understanding of the pathogenesis of inflammation.

THP-1 cells, a human monocytic leukemia cell line, have been extensively employed as a model for examining the functional diversity of monocyte/macrophage transition, due to the similarities in biological behavior of monocytes/macrophages derived from peripheral blood and the fact that non-adherent cells can be changed into plastic-adherent macrophages by $\alpha_{\mathrm{V}} \beta_{3}$ integrin expression via ERK activation in the presence of phorbol myristate acetate (PMA) $(10,11)$.

In the present study, we investigated the effects of IFN- $\gamma$ on cell adhesion and the production of tumor necrosis factor (TNF) using PMA-stimulated THP-1 cells.

\section{Materials and methods}

Reagents. PMA was purchased from Sigma (St. Louis, MO, USA). IFN- $\gamma$ was obtained from Peprotech EC, Ltd. (London, UK). U0126 (MEK inhibitor) and BAY 11-7082 (I $\mathrm{B}$ inhibitor) were obtained from Calbiochem (La Jolla, CA, USA). Fluorescein isothiocyanate (FITC)-conjugated primary antibodies for the flow cytometric assay were as follows: $\alpha_{\mathrm{V}}$ integrin, $\beta_{3}$ integrin (BioLegend, San Diego, CA); intercellular adhesion molecule-1 (ICAM-1) (Immunotech, Marseille, France), vascular cell adhesion molecule-1 (VCAM-1) (R\&D Systems, Minneapolis, MN, USA) and isotype IgG (BD Biosciences Pharmingen, San Diego, CA). Antibodies for western blot analysis were as follows: anti-phospho-extracellular signal-regulated kinase (ERK)1/2, phospho-NF- $\kappa \mathrm{B}$ p65, anti-ERK1/2 and anti-NF- $\mathrm{B}$ p65 (Cell Signaling Technology, Beverly, MA, USA). Blocking antibodies were as follows: $\alpha_{\mathrm{V}}$ integrin (Abcam, Cambridge, MA, USA), ICAM-1, VCAM-1, IFN- $\gamma$ R and isotype IgG1 (R\&D Systems). Reagents for real-time PCR were as follows: RNeasy Mini kit (Qiagen, 
Table I. Primers used for RT-qPCR.

\begin{tabular}{lll}
\hline Gene & \multicolumn{1}{c}{ Forward } & Reverse \\
\hline GAPDH & GCACCGTCAAGGCTGAGAAC & TGGTGAAGACGCCAGTGGA \\
TNF & GTGACAAGCCTGTAGCCCATGTT & TTATCTCTCAGCTCCACGCCATT \\
ICAM-1 & AACTGACACCTTTGTTAGCCACCTC & CCCAGTGAAATGCAAACAGGAC \\
VCAM-1 & CGAAAGGCCCAGTTGAAGGA & GAGCACGAGAAGCTCAGGAGAAA \\
Integrin $\alpha_{\mathrm{V}}$ & GGAGCAATTCGACGAGCACT & TTCATCCCGCAGATACGCTA \\
Integrin $\beta_{3}$ & TGACGAAAATACCTGCAACCG & GCATCCTTGCCAGTGTCCTTAA
\end{tabular}

RT-qPCR, reverse transcription-quantitative polymerase chain reaction; GAPDH, glyceraldehyde 3-phosphate dehydrogenase; TNF, tumor necrosis factor; ICAM-1, intercellular adhesion molecule 1; VCAM-1, vascular cell adhesion molecule 1.

Valencia, CA, USA), PrimeScript RT Reagent kit (Takara Bio Inc., Shiga, Japan) and Thunderbird qPCR mix (Toyobo, Osaka, Japan). Primers were produced by Takara Bio (Table I), with the exception of the $\alpha_{\mathrm{V}}$ and $\beta_{3}$ integrins, which were produced by Invitrogen (Carlsbad, CA, USA).

Cell culture. Human monocytic THP-1 cells (American Type Culture Collection, Manassas, VA, USA) were cultured in RPMI-1640 medium supplemented with 5\% fetal bovine serum (Gibco, Los Angeles, CA, USA), 1\% penicillin/streptomycin and $1 \% \mathrm{~L}$-glutamin at $37^{\circ} \mathrm{C}$ in a humidified $5 \% \mathrm{CO}_{2}$ atmosphere.

Cell attachment assay. The cell attachment assay was performed as described previously (11). THP-1 cells $\left(2 \times 10^{5} / \mathrm{ml}\right)$ were incubated in uncoated 96 -well plates for $\sim 48 \mathrm{~h}$ following PMA stimulation in the presence or absence of IFN- $\gamma$. Total (without washing) and adherent (with washing) cell numbers were measured by adding the cell count reagent (Nacalai Tesque, Kyoto, Japan). Following $3 \mathrm{~h}$ of incubation, absorbance at $450 \mathrm{~nm}$ was calculated using FlexStation 3 (Molecular Devices, Sunnyvale, CA, USA). Pretreatment with inhibitors (U0126 and BAY 11-7082) or blocking antibodies was performed for 30 min prior to the addition of PMA with or without IFN- $\gamma$, followed by $48 \mathrm{~h}$ of incubation and the obtained data were expressed as the relative adherent cell number (O.D.).

Western blot analysis. THP-1 cells were incubated in a $100 \mathrm{~mm}$ dish for up to $48 \mathrm{~h}$ after PMA stimulation in the presence or absence of IFN- $\gamma$. Cell extracts were obtained for western blot analysis as previously described (11). Antibodies were diluted in Hikari (Nacalai) signal enhancer for the detection of phosphorylated protein. Immunostained blots were stripped with Restore (Thermo Fisher Scientific, San Jose, CA, USA) and reprobed with primary antibodies against total protein. LAS-4000 and MultiGauge software (Fujifilm, Tokyo, Japan) were used for analysis.

Flow cytometric analysis. THP-1 cells were incubated in a $100 \mathrm{~mm}$ dish for $48 \mathrm{~h}$ following stimulation with PMA in the presence or absence of IFN- $\gamma$. Adherent cells were detached by treatment with trypsin/EDTA, washed with PBS and the non-adherent cells and incubated for $20 \mathrm{~min}$ with primary antibody, followed by fixation with $1 \%$ formaldehyde in
PBS. The expression of cell surface adhesion molecules was determined using flow cytometric analysis (FACScan, Becton Dickinson, Franklin Lakes, NJ, USA) and the data were analyzed using FlowJo (Tree Star, Inc., Ashland, OR, USA). As described previously (11), the mean fluorescence intensity (MFI) ratio is defined as MFI of the sample (adhesion molecule)/MFI of isotype IgG. The obtained data were expressed as the relative MFI ratio, and evaluated by comparing the MFI ratio.

Real-time reverse transcription-quantitative polymerase chain reaction ( $R T-q P C R)$. THP-1 cells were cultured in 6 or 12 -well plates for up to $24 \mathrm{~h}$. Following incubation, total RNA extraction, the reverse transcriptase reaction and real-time PCR were performed as described previously (11). Relative gene expression was analyzed using the $2^{-\Delta \Delta C T}$ method. In the inhibitor and blocking antibody study, the data were shown as relative to PMA single treatment.

ELISA. THP-1 cells were cultured with PMA stimulation in the presence or absence of IFN- $\gamma$ for up to $48 \mathrm{~h}$ without serum starvation treatment. The concentration of TNF in the supernatant was measured using an ELISA kit (R\&D Systems). The cell number, measured by hemocytometer, was used as a control for variability.

Statistical analysis. The data were analyzed by Prism (www.graphpad.com). Comparison between the groups was performed using one-way ANOVA and the unpaired t-test. Newman-Keuls post hoc test was used for the ANOVA. $\mathrm{P}<0.05$ was deemed to indicate a statistically significant difference.

\section{Results}

IFN- $\gamma$ augments PMA-induced cell attachment and TNF production. In the colorimetric adhesion assay, IFN- $\gamma$ did not induce cell attachment, whereas it dose-dependently upregulated adhesion in the presence of PMA (Fig. 1A). Similarly, IFN- $\gamma$ elevated the production of TNF at the mRNA and protein levels in the presence of PMA (Fig. 1B and C). The enhancing effect of IFN- $\gamma$ on cell attachment and TNF production was eliminated by the blocking antibody for the IFN- $\gamma$ receptor, confirming its receptor-mediated action (Fig. 1E and F). 
A

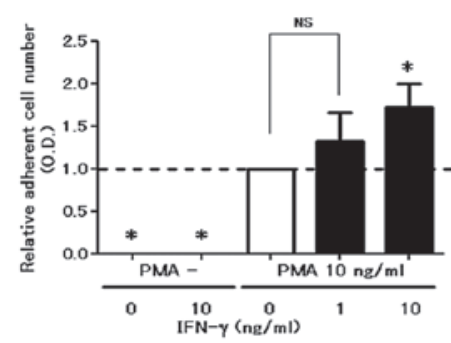

B

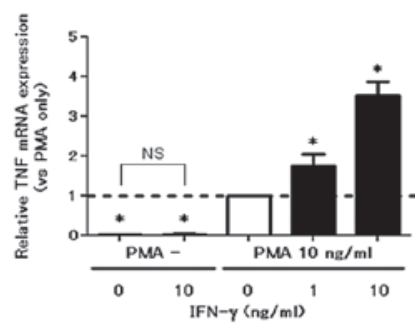

C

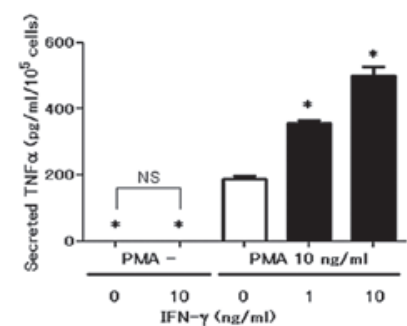

D

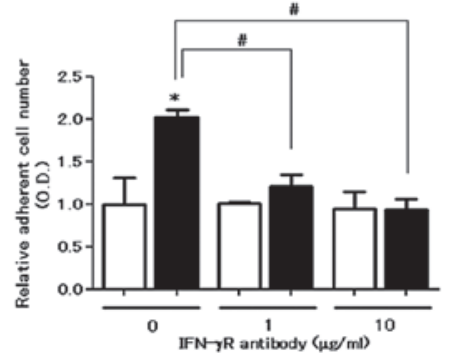

E

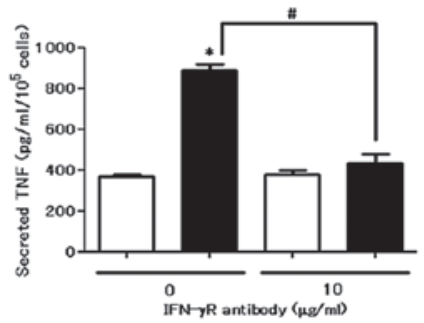

Figure 1. THP-1 cells were incubated in the presence of PMA $(10 \mathrm{ng} / \mathrm{ml})$ and IFN- $\gamma(10 \mathrm{ng} / \mathrm{ml})$ for up to $48 \mathrm{~h}$. (A-C) The effect of IFN- $\gamma$ addition on the relative adherent cell number (O.D.), relative TNF mRNA expression and secretion. (D and E) Anti-IFN- $\gamma$ R antibody blocked the enhancing effect of PMA and IFN- $\gamma$ induced cell attachment and TNF production. The data show the means \pm SD of three samples from one experiment performed at least three times separately. ${ }^{*} \mathrm{P}<0.05$ vs. PMA only, ${ }^{\sharp} \mathrm{P}<0.05$ vs. PMA and IFN- $\gamma$. PMA, phorbol myristate acetate; IFN- $\gamma$, interferon- $\gamma$; TNF, tumor necrosis factor.

A

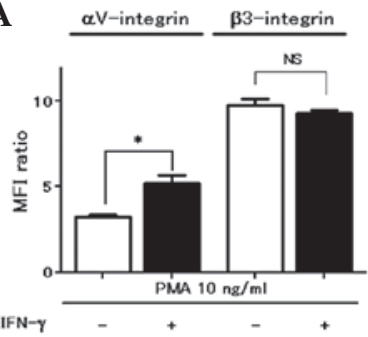

C

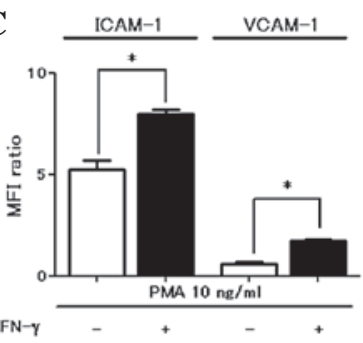

B

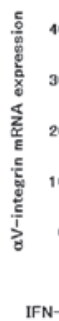

D

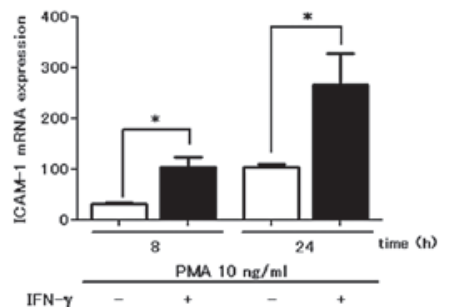

aV-integrin

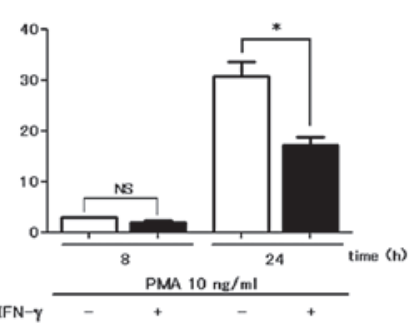

ICAM-1
$\mathbf{E}$

E

$\mathbf{F}$
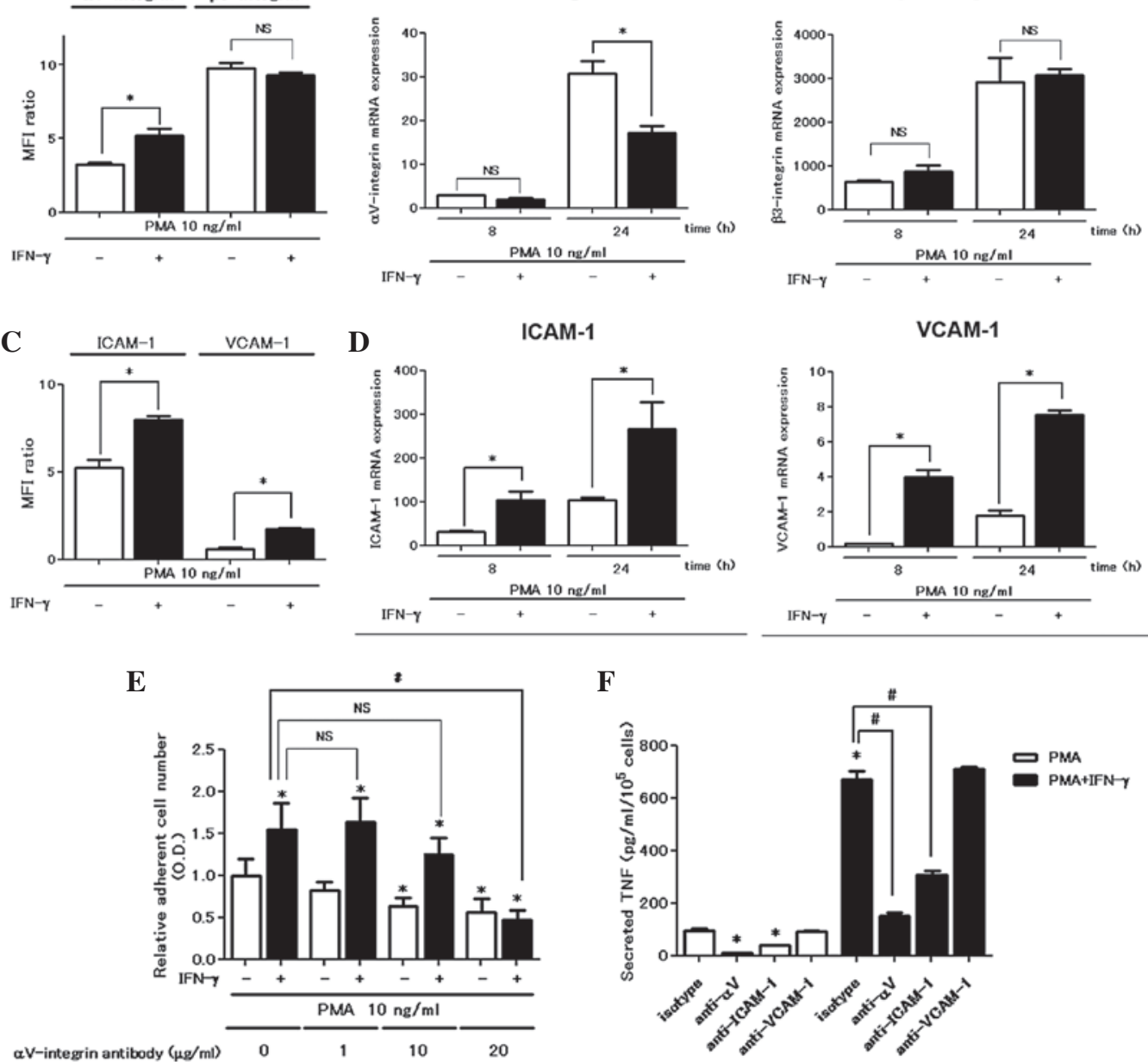

VCAM-1
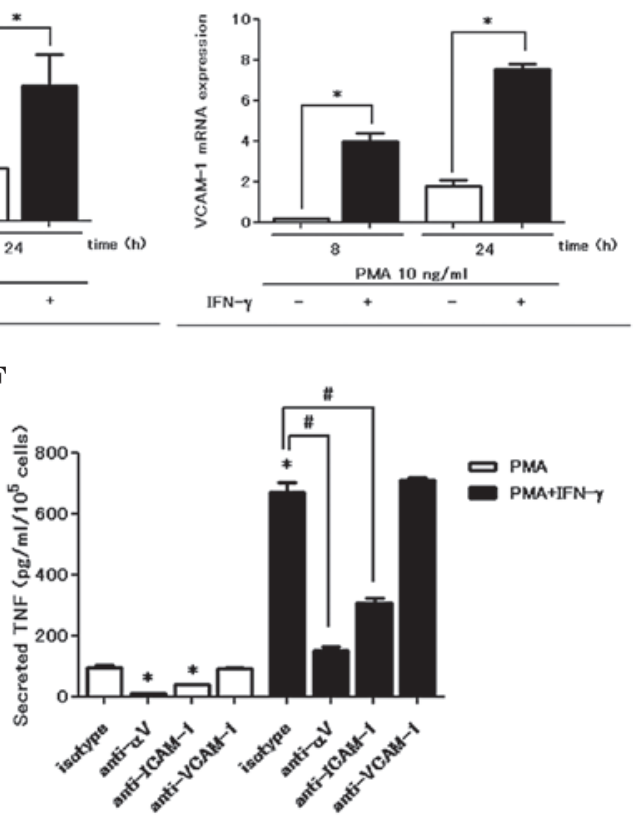

Figure 2. The effect of the addition of IFN- $\gamma$ on adhesion molecule mRNA and protein levels. (A and B) Flow cytometry and RT-qPCR of the $\alpha_{\mathrm{V}}$ and $\beta_{3}$ integrins. (C and D) Flow cytometry and RT-qPCR of ICAM-1 and VCAM-1. (E) Anti- $\alpha_{\mathrm{V}}$ integrin blocking antibody inhibited PMA with or without IFN- $\gamma$-induced cell attachment. (F) Anti- $\alpha_{\mathrm{V}}$ integrin and anti-ICAM-1 blocking antibody inhibited PMA with or without IFN- $\gamma$-induced TNF production. The data show the means \pm SD of three samples from one experiment performed at least three times separately. ${ }^{*} \mathrm{P}<0.05$ vs. PMA only, ${ }^{\#}<<0.05$ vs. PMA and IFN- $\gamma$. PMA, phorbol myristate acetate; IFN- $\gamma$, interferon- $\gamma$; TNF, tumor necrosis factor. 

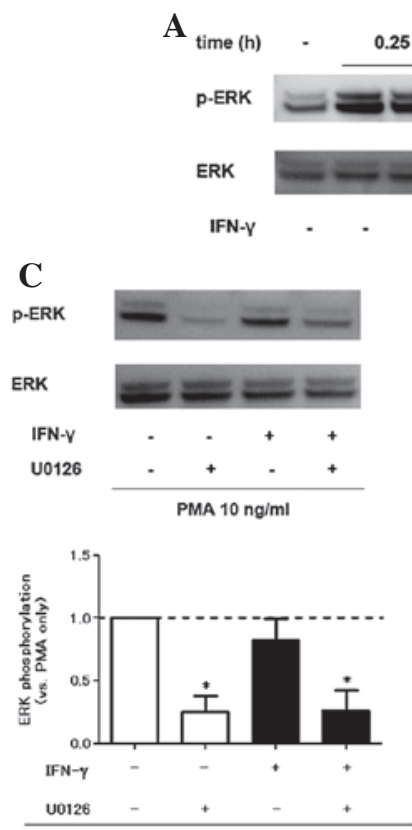

D
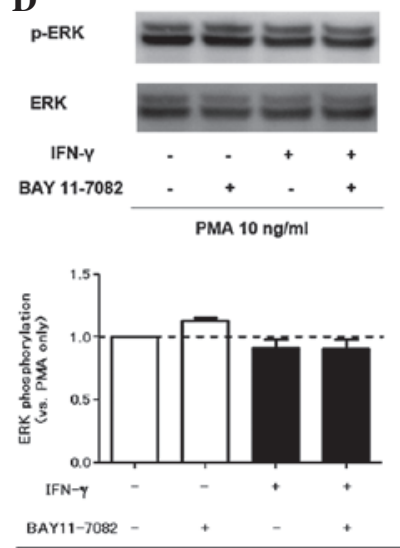

B

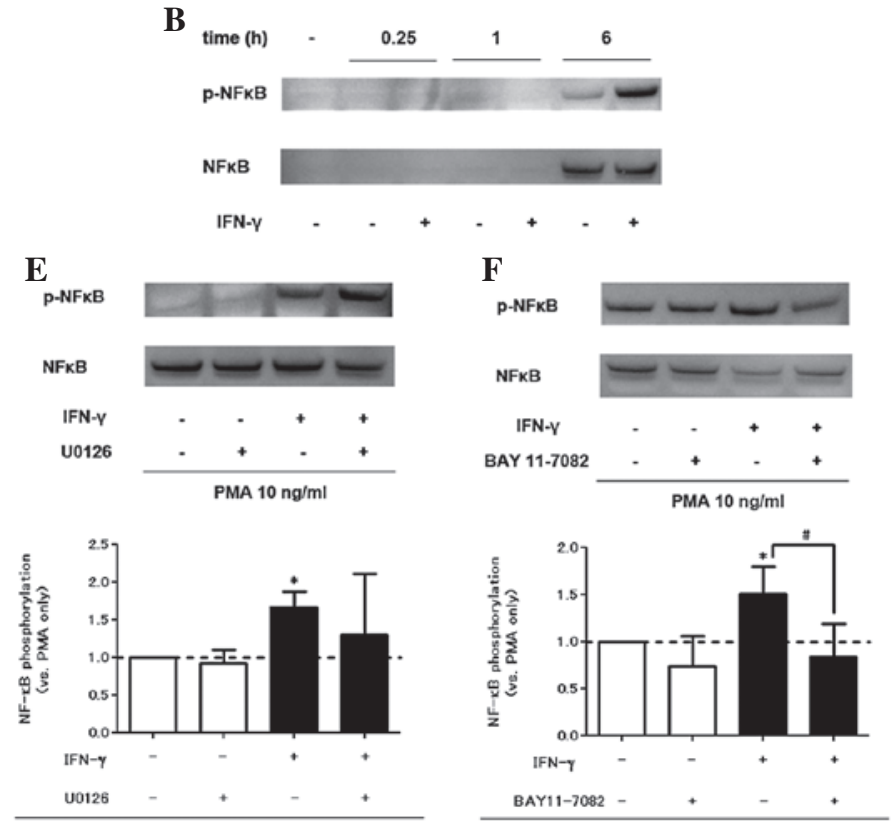

$\mathbf{E}$

$\mathbf{E}_{\mathrm{P}-\mathrm{NFKB}}$

Figure 3. (A and B) The effect of IFN- $\gamma$ on PMA-induced ERK and NF-kB phosphorylation in western blot analysis. (C-F) The inhibitory effect of U0126 $(10 \mu \mathrm{M})$ and BAY 11-7082 $(1 \mu \mathrm{M})$ on ERK and NF- $\mathrm{B}$ phosphorylation. The data show the means \pm SD of three samples from one experiment performed at least three times separately. $\mathrm{P}<0.05$ vs. PMA only, ${ }^{"} \mathrm{P}<0.05$ vs. PMA and IFN $-\gamma$. PMA, phorbol myristate acetate; IFN- $\gamma$, interferon- $\gamma$.
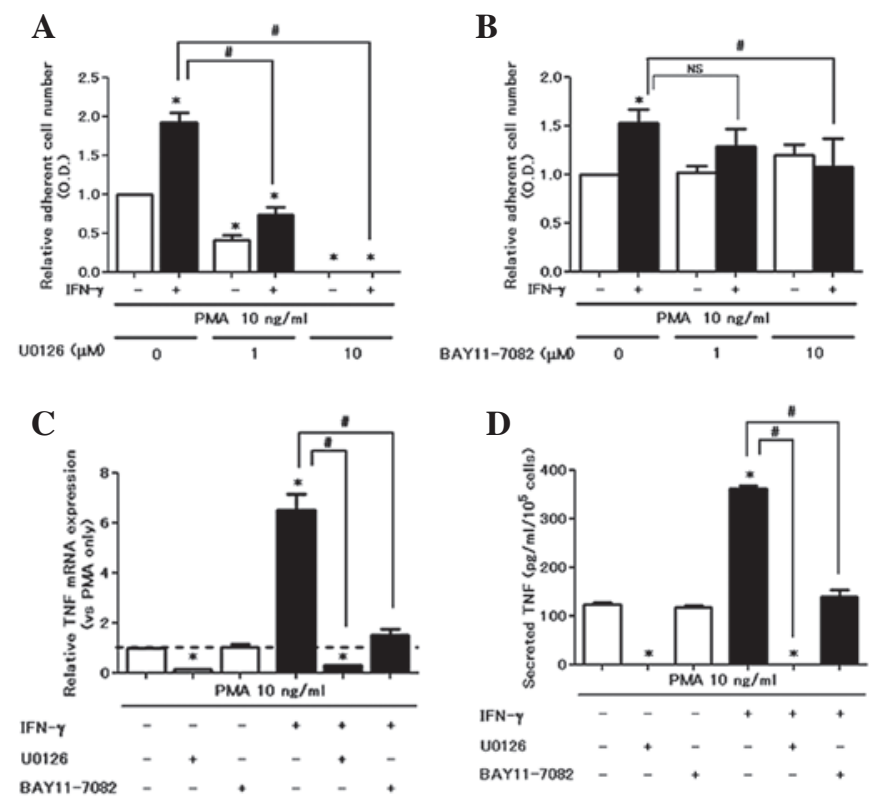

Figure 4. (A) Pretreatment with U0126 (10 $\mu \mathrm{M})$ inhibited PMA-induced basal and IFN- $\gamma$ enhanced cell attachment. (B) Pretreatment with BAY 11-7082 $(1 \mu \mathrm{M})$ inhibited the effect of IFN- $\gamma$ on cell adhesion. (C and D) The inhibitory effect of U0126 and BAY 11-7082 on relative TNF mRNA expression and secretion. The data show the means \pm SD of three samples from one experiment performed at least three times separately. $\mathrm{P}<0.05$ vs. PMA only, ${ }^{\text {}} \mathrm{P}<0.05$ vs. PMA and IFN- $\gamma$. PMA, phorbol myristate acetate; IFN- $\gamma$, interferon- $\gamma$; TNF, tumor necrosis factor.

The $\alpha_{V}$ integrin is involved in the enhancing effects of IFN- $\gamma$ on cell attachment and TNF production. We examined the expression of cell surface adhesion molecules using a flow cytometric assay. IFN- $\gamma$ enhanced cell-surface $\alpha_{\mathrm{V}}$ integrin expression in the flow cytometric assay; however, it did not increase either $\alpha_{\mathrm{V}}$ integrin mRNA or $\beta_{3}$ integrin expression levels (Fig. 2A and B). IFN- $\gamma$ was active in upregulating the expression of other adhesion molecules such as ICAM-1 and VCAM-1 at the mRNA and protein levels (Fig. 2C and D). Pretreatment with the $\alpha_{\mathrm{V}}$ integrin blocking antibody $(10 \mu \mathrm{g} / \mathrm{ml})$ significantly inhibited PMA-stimulated cell attachment, confirming the involvement of the $\alpha_{\mathrm{V}}$ integrin in PMA-induced plastic adhesion and a higher concentration $(20 \mu \mathrm{g} / \mathrm{ml})$ of the blocking antibody was required in order to inhibit the cell attachment enhanced by treatment with IFN- $\gamma$ (Fig. 2E). However, the blocking antibodies for ICAM-1 and VCAM-1 did not inhibit PMA with or without IFN- $\gamma$-induced cell attachment even at higher concentrations (data not shown). Furthermore, treatment with the blocking antibody for ICAM-1 suppressed PMA with or without IFN- $\gamma$-induced TNF secretion (Fig. 2F).

IFN- $\gamma$ treatment augments $N F-\kappa B$ phosphorylation but not ERK phosphorylation. We analyzed the PMA and IFN- $\gamma$-induced phosphorylated proteins in the signaling pathways of inflammation. In western blot analysis, PMA alone induced ERK and NF- $\kappa \mathrm{B}$ phosphorylation. Addition of IFN- $\gamma$ also enhanced NF- $\kappa \mathrm{B}$ phosphorylation, but not ERK phosphorylation (Fig. 3A and B). The specific inhibitory effect of U0126 and BAY 11-7082 on the signaling pathways was confirmed (Fig. 3C-F). As for viability, pretreatment with signaling pathway inhibitors did not affect cell viability in the trypan blue exclusion assay within the range of concentrations used in the experiment (data not shown).

IFN- $\gamma$ amplifies PMA-induced cell attachment and TNF production via the $N F-\kappa B$ pathway. We examined the effect of inhibitors on cell attachment and TNF production from PMA with or without IFN- $\gamma$-treated cells. With regard to cell 
attachment, U0126 (MEK inhibitor) completely inhibited PMA \pm IFN- $\gamma$-induced cell attachment in a dose-dependent manner (Fig. 4A). However, BAY 11-7082 counteracted the IFN- $\gamma$-induced cell attachment increase (Fig. 4B), but did not inhibit PMA-induced cell attachment. U0126 almost completely eliminated the production of TNF in PMA with or without IFN- $\gamma$-stimulated cells at mRNA and protein levels (Fig. 4C and 4D). By contrast, BAY 11-7082 enhanced the production of TNF in PMA with a single treatment. However, BAY 11-7082 significantly suppressed IFN- $\gamma$-enhanced TNF production (Fig. 4C and D). These data indicate that the ERK pathway is critical for mediating PMA-induced baseline cell activation and that an increase by IFN- $\gamma$ is dependent on the NF-кB pathway.

\section{Discussion}

IFN- $\gamma$ induces adhesion molecule expression, amplifies inflammatory cytokine production and promotes monocyte/ macrophage antimicrobial and antitumor activity (12). The enhancement of lipopolysaccharide (LPS) responses by IFN- $\gamma$ is known as a priming effect (13). However, the mechanism involved in macrophage adhesion and cytokine production amplification remains unclear. In this study, we revealed the pivotal role of the NF- $\kappa$ B pathway in mediating the upregulation of IFN- $\gamma$ using PMA-induced THP-1 cell adhesion and a TNF secretion assay.

The presence of a non-absorbable foreign body induces persistent inflammation, known as a foreign body reaction, in vivo, although biomaterial surface properties are important in modulating its reaction $(14,15)$. The importance of IFN- $\gamma$ in the foreign body reaction is indicated in an animal study (16) and IFN- $\gamma$ appears to be a crucial cytokine in granuloma formation as it accelerates their various functions, including their adhesive properties.

After treatment with PMA, the non-adherent THP-1 monocytic cells became adherent to plastic, which was blocked by $\alpha_{V}$ integrin function-blocking antibody as described in a previous study (11). In the present study, IFN- $\gamma$ upregulated cell adhesion and cell surface $\alpha_{\mathrm{v}}$ integrin expression in the presence of PMA. Accordingly, sufficient concentration of $\alpha_{v}$ integrin blocking antibody was acquired for the inhibition of IFN- $\gamma$-enhanced adhesion. Blocking antibodies for other adhesion molecules with the immunoglobulin superfamily, such as ICAM-1 and VCAM-1, which were also upregulated by IFN- $\gamma$ treatment, failed to inhibit the amplification of cell adherence. Qualitative alteration of adhesion molecules, including increased avidity, may also be involved in the enhancement of adhesive properties, since the upregulation of the $\alpha_{\mathrm{V}}$ integrin protein was not accompanied by mRNA expression. In contrast to cell adhesion, the ICAM-1 blocking antibody inhibited the production of TNF. The difference in effectiveness between the $\alpha_{\mathrm{V}}$ integrin blocking antibody and the ICAM-1 blocking antibody against cell adhesion is due to the different mechanisms involved in cell adhesion; the former is mainly involved in cell-matrix adhesion and the latter is involved in cell-cell adhesion.

The ERK signaling pathway is critical for PMA-induced cell adherence and TNF secretion. PMA treatment induced ERK phosphorylation and subsequent cell adhesion and
TNF expression. U0126 (MEK inhibitor) almost completely eliminated PMA-induced adhesion and TNF production, a result that is in agreement with previous observations (11). Even in co-stimulation with PMA and IFN- $\gamma$, U0126 delivered a profound inhibition of adhesion and TNF production, emphasizing the fundamental role of the ERK pathway in macrophage function.

$\mathrm{NF}-\kappa \mathrm{B}$ appears to be important in mediating IFN- $\gamma$ bioactivity. IFN- $\gamma$ enhanced the phosphorylation of NF- $\mathrm{KB}$ without any effect on ERK phosphorylation. In addition, BAY 11-7082 was an effective inhibitor of the IFN- $\gamma$-induced enhancing action on adhesion and TNF production. BAY 11-7082 has been widely used as an anti-inflammatory agent in previous studies $(17,18)$ and our results support its inhibitory effects on IFN- $\gamma$-enhancing action in adhesion and TNF production by macrophages.

In conclusion, we revealed a close correlation between IFN- $\gamma$ action and the NF- $\mathrm{BB}$ pathway with regards to PMA-induced THP-1 cell adhesion and TNF production, which explains, in part, the process by which IFN- $\gamma$ accelerates the inflammatory process.

\section{Acknowledgements}

We are grateful for the technical support we received from the Research Support Center at Kyushu University's Graduate School of Medical Sciences. This study was in part supported by grants from the Ministry of Health, Labour and Welfare, and the Ministry of Education, Culture, Sports, Science and Technology, Japan.

\section{References}

1. Takeda K, Ichiki T, Narabayashi E, et al: Inhibition of prolyl hydroxylase domain-containing protein suppressed lipopolysaccharide-induced TNF- $\alpha$ expression. Arterioscler Thromb Vasc Biol 29: 2132-2137, 2009.

2. Allison AC, Ferluga $\mathrm{H}$ and Schorlemmer HU: The role of macrophage activation in chronic inflammation. Agents Actions 8: 27-35, 1978.

3. Sorg C: Macrophages in acute and chronic inflammation. Chest 100: 173S-175S, 1991

4. Todd RF III, Alvarez PA, Brott DA and Liu DY: Bacterial lipopolysaccharide, phorbol myristate acetate, and muramyl dipeptide stimulate the expression of a human monocyte surface antigen, Mo3e. J Immunol 135: 3869-3877, 1985.

5. Schroder K, Hertzog PJ, Ravasi T and Hume DA: Interferon- $\gamma$ : an overview of signals, mechanisms and functions. J Leukoc Biol 75: 163-189, 2004.

6. Scheibenbogen C and Andreesen R: Development regulation of the cytokine repertoire in human macrophages: IL-1, IL-6, TNF- $\alpha$, and M-CSF. J Leukoc Biol 50: 35-42, 1991.

7. Cassatella MA, Bazzoni F, Flynn RM, Dusi S, Trinchieri G and Rossi F: Molecular basis of interferon- $\gamma$ and lipopolysaccharide enhancement of phagocyte respiratory burst capability. Studies on the gene expression of several NADPH oxidase components. J Biol Chem 265: 20241-20246, 1990.

8. Moller DR: Cells and cytokines involved in the pathogenesis of sarcoidosis. Sarcoidosis Vasc Diffuse Lung Dis 16: 24-31, 1999.

9. Sasaki M, Namioka Y, Ito T, et al: Role of ICAM-1 in the aggregation and adhesion of human alveolar macrophages in response to TNF- $\alpha$ and IFN- $\gamma$. Mediators Inflamm 10: 309-313, 2001.

10. Chanput W, Mes J, Vreeburg RA, Savelkoul HF and Wichers HJ: Trascription profiles of LPS-stimulated THP-1 monocytes and macrophages: a tool to study inflammation modulating effects of food-derived compounds. Food Funct 1: 254-261, 2010 . 
11. Kurihara Y, Nakahara T and Furue M: $\alpha_{\mathrm{y}} \beta_{3}$-integrin expression through ERK activation mediates cell attachment and is necessary for production of tumor necrosis factor alpha in monocytic THP-1 cells stimulated by phorbol myristate acetate. Cell Immunol 270: 25-31, 2011.

12. Kimball ES, Kovacs E, Clark MC and Schneider CR: Activation of cytokine production and adhesion molecule on THP-1 myelomonocytic cells by macrophage colony-stimulating factor in combination with interferon- $\gamma$. J Leukoc Biol 58: 585-594, 1995.

13. Hayes MP, Freeman SL and Donnelly RP: IFN- $\gamma$ priming of monocytes enhances LPS-induced TNF production by augmenting both transcription and mRNA stability. Cytokine 7: 427-435, 1995 .

14. Klinge U, Theuer S, Krott E and Fiebeler A: Absence of circulating aldosterone attenuates foreign body reaction around surgical sutures. Lagenbecks Arch Surg 395: 429-435, 2010.
15. Anderson JM, Rodriguez A and Chang DT: Foreign body reaction to biomaterials. Semin Immunol 20: 86-100, 2008.

16. Khouw IM, van Wachem PB, van der Worp RJ, van den Berg TK, de Leij LF and van Luyn MJ: Systemic anti-IFN- $\gamma$ treatment and role of macrophage subsets in the foreign body reaction to dermal sheep collagen in rats. J Biomed Mater Res 49: 297-304, 2000.

17. Juliana C, Fernandes-Alnemri T, Wu J, et al: Anti-inflammatory compounds parthenolide and Bay 11-7082 are direct inhibitors of the inflammasome. J Biol Chem 285: 9792-9802, 2010.

18. Zhu B, Liu Z, Wang P, Wu C and Xu H: A nuclear factor-kappaB inhibitor BAY11-7082 inhibits interaction between human endothelial cells, T cells and monocytes. Transplant Proc 40: 2724-2728, 2008. 